EPJ Web of Conferences 72, 00003 (2014)

DOI: $10.1051 /$ epjconf / 20147200003

(C) Owned by the authors, published by EDP Sciences, 2014

\title{
Physics with KLOE and KLOE-2
}

\author{
Fabio Bossi ${ }^{1, *}$ for the KLOE/KLOE-2 Collaborations ${ }^{\dagger}$ \\ ${ }^{1}$ Laboratori Nazionali dell'INFN Frascati
}

\begin{abstract}
During the last 10 years, the KLOE experiment of the Frascati National Laboratories of INFN has produced many important results in the fields of flavour physics, hadron interactions and tests of the Standard Model. The analysis of the data taken between years 2000 and 2006 is still undergoing. A new data taking run with an improved detector is expected to start by beginning of 2014. Its perspectives will be briefly mentioned.
\end{abstract}

*e-mail:fabio.bossi@lnf.infn.it

$\dagger$ KLOE-2 collaboration: D. Babusci, I. Balwierz-Pytko, G. Bencivenni, C. Bloise, F. Bossi, P. Branchini, A. Budano, L. Caldeira Balkestảhl, G. Capon, F. Ceradini, P. Ciambrone, F. Curciarello, E. Czerwiński, E. Danè, V. De Leo, E. De Lucia, G. De Robertis, A. De Santis, P. De Simone, A. Di Cicco, A. Di Domenico, C. Di Donato, R. Di Salvo, D. Domenici, O. Erriquez, G. Fanizzi, A. Fantini, G. Felici, S. Fiore, P. Franzini, A. Gajos, P. Gauzzi, G. Giardina, S. Giovannella, E. Graziani, F. Happacher, L. Heijkenskjöld B. Höistad, M. Jacewicz, T. Johansson, K. Kacprzak, D. Kamiǹska, A. Kupsc, J. Lee-Franzini, F. Loddo, S. Loffredo, G. Mandaglio, M. Martemianov, M. Martini, M. Mascolo, R. Messi, S. Miscetti, G. Morello, D. Moricciani, P. Moskal, F. Nguyen, A. Palladino, A. Passeri, V. Patera, I. Prado Longhi, A. Ranieri, P. Santangelo, I. Sarra, M. Schioppa, B. Sciascia, M. Silarski, C. Taccini, L. Tortora, G. Venanzoni, W. Wis̀licki, M. Wolke, J. Zdebik.

This is an Open Access article distributed under the terms of the Creative Commons Attribution License 4.0, which permits unrestricted use, distribution, and reproduction in any medium, provided the original work is properly cited. 


\section{The past and the present: KLOE}

From year 2000 to 2006, the KLOE experiment has acquired data at the DAФNE $e^{+} e^{-}$collider of the Frascati National Laboratories of INFN (LNF). During this period, a record integrated luminosity of $\sim 2.5 \mathrm{fb}^{-1}$ has been collected at a center of mass energy of $1020 \mathrm{MeV}$, corresponding to the mass of the $\phi$ meson, as well as other $\sim 240 \mathrm{pb}^{-1}$ at $1000 \mathrm{MeV}$. This corresponds to an increase of about two orders of magnitude with respect to what collected by previous experiments at running the same energy.

The $\phi$ meson decays predominantly into a pair of charged or neutral kaons, but also, with lower branching ratios, to 3 pions or radiatively into light scalar or pseudoscalar mesons.

The main advantage of a $\phi$-factory such as DAФNE rests in the absolute cleanliness of the final states, which can be completely reconstructed by a $4 \pi$ detector such as KLOE. Very often, one has simple means to tag by first principles the presence of a well defined type of particle (a kaon or a $\eta$ meson, for instance) with a given momentum. Also, in the case of the decay into neutral kaons, the two final state particles are quantumly correlated, which allows one performing subtle and unique tests of quantum interferometry.

Thanks to these features, and to the huge collected statistics, KLOE has produced in the course of the last ten years several important results in many different fields, including:

- The complete set of measurements, using charged and neutral kaons decays, needed to perform the best determination of the CKM matrix element $\mathrm{V}_{u s}$. This, in turn, has allowed performing the best test to date of the unitarity of this matrix, with a precision better than one part in ten thousands.

- Several independent determinations of the hadronic contribution to the muon anomaly $a_{\mu}$, the only part which cannot be computed analytically. As it is well known, this result, combined with theoretical computations, points to a more than $3 \sigma$ discrepancy between the measured and theoretical values of $a_{\mu}$. At present this is the largest discrepancy between an experiment and Standard Model expectations.

- Several different tests of discrete symmetries conservation in fundamental interactions, using kaon and $\eta$ meson decays.

- Tests of low-energy QCD, including studies on the nature of the scalar mesons $\mathrm{a}_{0}$ and $\mathrm{f}_{0}$.

- Searches for signals of New Physics, in particular of the existence of non-standard light neutral vector bosons, the so called " $U$ bosons" or "dark photons". No positive signal has been observed to date, thus limits on its existence have been set.

The analysis of this enormous harvest of data is still going on. Some of the most recent physics results are the object of the contribution to this Conference by Curciarello, De Leo, Di Domenico and Sarra. I will not spend more words on that. I will concentrate in the following onto the future of the experiment which is being pursued under the name of KLOE-2.

\section{The future: KLOE-2}

During years 2008-2009, the Accelerator Division of LNF has tested a new interaction scheme on DAФNE, the so called "crab-waist" scheme, with the hope of increasing its luminosity, other things being equal. The experiment, performed while running for the non-magnetic detector SIDDHARTA, was succesful, reaching a peak luminosity larger by a factor $\sim 3$ with respect to the best observed with KLOE in 2005 [1]. Therefore it was natural to propose the implementation of the new scheme also with KLOE, for a new data taking run.

Some detector modification were at the same time proposed. Actually, the original KLOE apparatus was composed by two main subdetectors: a very large cylindrical drift chamber and a hermetic lead-scintillating fibers electromagnetic calorimeter with exceptional timing performance. The design and the dimensions of these detectors were motivated mainly by the 
need for optimizing the observation of the decay products of long-lived neutral kaons, whose mean decay path is of $3.5 \mathrm{~m}$ at DAФNE energies. On the other hand, the new data taking will concentrate mostly on the physics produced close to the interaction point [2]. In order to optimize efficiency for these specific topics, three new subdetectors have been designed and built in the course of the last three years: an internal tracker, with the aim of improving the resolution for charged vertices produced close to the interaction point [3]; two different type of calorimeters, to improve detection of photons emitted at very low polar angles or hitting the low- $\beta$ insertion quadrupoles of the machine [4] [5].

While the two calorimeters are detectors of relatively standard concept, the inner tracker design follows a totally new idea, that of a cylindrical GEM detector. In fact, before KLOE-2, GEM detectors were only built in a planar geometry. A long prototyping work has been carried out in Frascati, by the group lead by G. Bencivenni. The final detector was then assembled and was ready for insertion by May 2013. Monte Carlo studies have shown that this four-layers detector can improve the resolution on charged vertices in the region interesting for quantum inteferometry by a factor of $\sim 3$.

Moreover, two pairs of dedicated tagging stations have been installed with the purpose of indentifying the scattered electron and positron from $\gamma-\gamma$ reactions, improving background recjection for this type of events. The two pair of stations are placed into totally different position (one inside, the other one outside the main detector), in order to cover two well separated regions of the spectrum of the scattered leptons.

All of the new subdetectors are now installed and operational and their debugging on collision data is expected to start very soon.

The goal of KLOE-2 is to collect a luminosity of $\sim 10 \mathrm{fb}^{-1}$ within the next three years. The physics goals of KLOE-2 are thoroughly discussed in [2]. Among the most important topics we can enumerate:

- Improvements in quantum interferometry. All of the measurements published so far by
KLOE are statistically limited, so acquiring new data will be beneficial. Moreover, as specified before, the insertion of the GEM tracker should improve also on systematics.

- Further searches for light neutral gauge bosons.

- Studies on very rare $K_{S}^{0}$ and $\eta$ decays.

- Studies on two photon interactions. In particular studies on the $\gamma \gamma \rightarrow \pi^{0}$ reaction, which is relevant for the determination of the light-bylight contribution to the muon anomaly

Despite some difficulties in the start-up of the machine, due mostly to failures of old hardware components, the first collisions with the KLOE magnet operational have been recently registered. Debugging of detector and machine is progressing well. We hope to start physics data taking soon.

\section{References}

[1] M. Zobov et al., Phys.Rev.Lett. 104174801 (2010)

[2] G. Amelino Camelia et al., Eur.Phys.J. C 68 619 (2010)

[3] A. Balla et al., Nucl.Instrum.Meth A 628 194 (2011)

[4] M. Cordelli et al., Nucl.Instrum.Meth A 617 105 (2010)

[5] F. Happacher et al., Nucl.Phys.Proc.Supp. 197215 (2009) 